\title{
Nanoporous silicon tubes: the role of geometry in nanostructure formation and application to light emitting diodes
}

\author{
Jelena Vukajlović Pleština ${ }^{1,4}$, Vedran Đerek ${ }^{2,3,4}$, Luca Francaviglia ${ }^{1}$, \\ Francesca Amaduzzi ${ }^{1}$, Heidi Potts ${ }^{1}$, Mile Ivanda ${ }^{2}$ \\ and Anna Fontcuberta i Morral ${ }^{1}$ \\ ${ }^{1}$ Laboratoire des Matériaux Semiconducteurs, École Polytechnique Fédérale de Lausanne, 1015 \\ Lausanne, Switzerland \\ ${ }^{2}$ Center of Excellence for Advanced Materials and Sensing Devices, Ruđer Bošković Institute, Zagreb \\ 10000, Croatia \\ ${ }^{3}$ Laboratory of Organic Electronics, Department of Science and Technology, Linköping University, \\ 60174 Norrköping, Sweden \\ E-mail: anna.fontcuberta-morral@epfl.ch and ivanda@irb.hr
}

Received 14 March 2017, revised 24 April 2017

Accepted for publication 8 May 2017

Published 8 June 2017

\begin{abstract}
Obtaining light emission from silicon has been the holy grail of optoelectronics over the last few decades. One of the most common methods for obtaining light emission from silicon is to reduce it to a nanoscale structure, for example by producing porous silicon. Here, we present a method for the large-area fabrication of porous silicon microtubes by the stain etching of silicon micropillar arrays. We explain and model how the formation of the microtubes is influenced by the morphology of the substrate, especially the concave or convex character of the 3D features. Light emission is demonstrated at the micro- and nanoscale respectively by photo- and cathodoluminescence. Finally, we demonstrate a $0.55 \mathrm{~cm}^{2}$ device that can work as a photodetector with $2.3 \%$ conversion efficiency under one sun illumination, and also as a broadband light emitting diode, illustrating the applicability of our results for optoelectronic applications.
\end{abstract}

Keywords: porous silicon, silicon photonics, light emitting diode, stain etching, micro nano fabrication, cathodoluminescence, luminescence

S Supplementary material for this article is available online

(Some figures may appear in colour only in the online journal)

\section{Introduction}

Silicon is the most widely used electronic material thanks to its abundance, functional properties and high-quality oxide. Despite many efforts in the last few decades, it has been challenging to obtain light emission from macroscopic silicon due to the indirect nature of its bandgap [1-7]. Nanoscale silicon is an efficient light emitter in the visible spectral range, although it is still far from demonstrating the efficiency of

\footnotetext{
${ }^{4}$ These authors contributed equally.
}

direct-bandgap semiconductors. One pathway of producing nanoscale silicon in high volumes is the utilization of porous silicon. The reported external efficiency of photoluminescence in porous silicon can be as high as $23 \%$, while electroluminescence efficiency stays at the $1 \%$ level [8-14]. The functional properties of silicon nanocrystals and porous silicon are thus encouraging for possible future use in photodetectors and emitters on a chip $[15,16]$.

Porous silicon can be generated by self-limited electrochemical etching in aqueous hydrofluoric acid (HF) or fluoride-containing solutions [17-21]. Besides direct anodization 


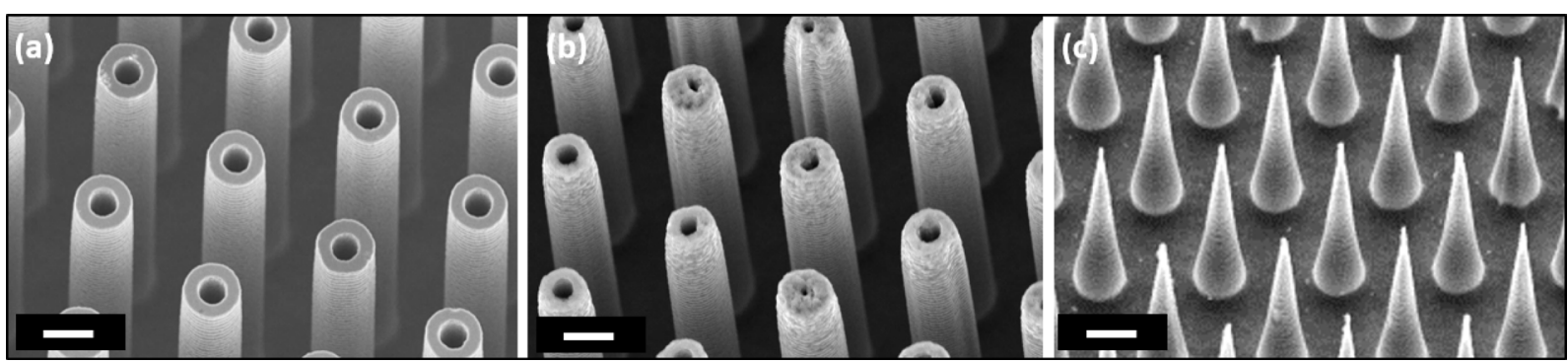

Figure 1. Different shapes obtained by stain etching: (a) Si tubes from etching in the BHNO solution, (b) silicon tubes covered by a layer of porous silicon from etching the tubes in the HVO solution and (c) silicon micro-cones covered by a layer of porous silicon; the scale bar is $2 \mu \mathrm{m}$.

in galvanostatic or potentiostatic conditions, it can also be prepared by electroless or stain etching. This technique involves an oxidant in an etchant agent, such as $\mathrm{V}_{2} \mathrm{O}_{5}$ in a fluoride solution. The oxidant provides additional holes to initiate the oxidation reactions necessary for the porous silicon formation. The use of $\mathrm{V}_{2} \mathrm{O}_{5}$ is preferred over $\mathrm{HNO}_{3}$ [22] because it avoids bubble formation, which affects the homogeneity of the layers [23, 24]. More details about the chemistry of stain etching using nitrates and the $\mathrm{V}_{2} \mathrm{O}_{5}$ used in this study can be found in the supplementary information (stacks.iop.org/JPhysD/50/265101/mmedia). Electroless etching has been widely used for the micro- and nanostructuring of planar silicon substrates, resulting in a roughened surface containing pits or porous structures [25, 26]. Such surfaces have been exploited for their highly exposed area [27, $28]$ and good light absorbing properties due to light trapping [29]. These porous structures contain silicon nanocrystals, which show efficient photoluminescence and electroluminescence, enabling optoelectronic and sensing applications [2937]. To the best of our knowledge, all examples of the solid state electroluminescent silicon devices presented so far are based on planar geometry [10, 33, 38-41].

The stain etching process depends strongly on the electrolyte composition, including the fluoride concentration and $\mathrm{pH}$, the oxidant type and concentration and the electrolyte temperature, as well as on the properties of the silicon substrate such as the dopant type, charge carrier concentration and crystalline orientation. Silicon dissolution in fluoride-containing electrolytes is primarily driven by the supply of holes in the silicon [42-44]. More details on the chemical reactions involved in stain etching can be found in the supplementary information (SI 2). Bringing the semiconductor and the electrolyte into contact with each other results in a redistribution of charges, as the Fermi level in the semiconductor and the redox level in the solution equalize. In the semiconductor, this results in band bending and thus the depletion or accumulation of carriers close to the surface. Depletion is accompanied by the formation of a space charge layer (SCR) containing fixed ionized dopants. In the electrolyte, this results in the formation of an interfacial electrical double layer [45]. The SCR layer is a major self-limiting mechanism in the dissolution of semiconductors, bringing a potential barrier into existence, which acts as a barrier for the charge transfer from the electrolyte to the silicon and vice versa [46]. In this sense, it can be used to avoid or facilitate dissolution in certain regions of the substrate and to create non-planar structures such as silicon microtubes [46, 47].
In this work, we exploit the effect of geometry in modifying the speed of the stain etching of Si micropillars as well as the engineering of the SCR for obtaining ordered arrays of a variety of shapes, including tubular structures with different porosity levels as well as very pointed cone structures. We elucidate the mechanism of tube formation and illustrate their optical properties by photoluminescence (PL) and cathodoluminescence (CL). Finally, we demonstrate one of the potential applications by presenting a functional, largearea 3D-structured light emitting diode and solar cell. This periodic tubular structure can find various applications in different fields starting with sensors [48], energy storage [49-51] and anodes in electrochemical batteries $[52,53]$ as well as in photonics $[54,55]$. The porous layer has an additional advantage in biological applications, such as in drug delivery [56], and the sharply pointed features are particularly interesting for applications in cell biology research $[57,58]$.

\section{The mechanism of nanoporous Si tube formation}

Silicon micropillar arrays formed by reactive ion etching were subjected to electroless stain etching in three different solutions: HNO (hydrofluoric acid, $\mathrm{HF}(48 \%)$ :nitric acid, $\mathrm{HNO}_{3}(70 \%)$ : surfactant $=2000: 2.5: 1$ ), BHNO (buffered HF (HF: $\left.\mathrm{NH}_{4} \mathrm{~F}=12.5 \%: 87.5 \%\right), \quad \mathrm{BHF}(7: 1): \mathrm{HNO}_{3}(70 \%)$ :surfac tant $=2000: 5: 1)$ and $\mathrm{HVO}\left(48 \% \mathrm{HF}\right.$ containing $0.05 \mathrm{M} \mathrm{l}^{-1}$ vanadium (V) oxide $\mathrm{V}_{2} \mathrm{O}_{5}$, which served as an oxidant). General-purpose nonionic fluorosurfactant Zonyl FS-300 was used as the surfactant. For more details about micropillar fabrication and the stain etching procedures see SI 1. Starting with the same geometry of pillars, but using different etching parameters (the doping level of $\mathrm{Si}$, the $\mathrm{pH}$ of the solutions and the etching time), we were able to obtain a variety of different structures. Figure 1 shows scanning electron micrographs (SEM) of the different shapes obtained by the stain etching of the Si micropillars under different conditions, using the BHNO or HVO solutions on different substrates and for different durations. We show examples of three different groups of features: microtubes with a very thin porous layer, figure 1(a), microtubes with a homogenous layer of nanostructured photo- and electro-luminescent porous silicon, figure 1(b), and pointed structures that are a consequence of the lateral etching of the pillars and tubes, figure 1(c). Further on in the text we will show more details on the porous structures and elucidate the underlying formation mechanism. 


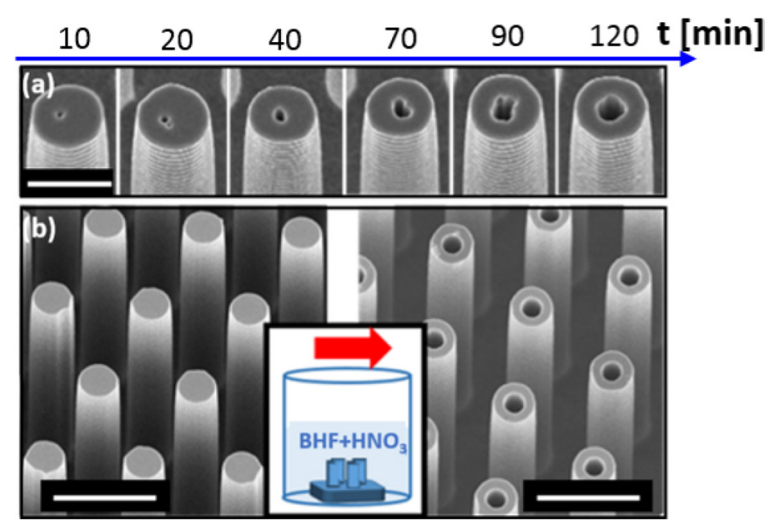

Figure 2. SEMs of the Si pillars upon etching in the BHNO solution. (a) Evolution of the morphology, (b) the original array and result after $150 \mathrm{~min}$ of etching.

We start by presenting the process of tube formation by looking at the evolution of the structures during stain etching. We use $36 \mu \mathrm{m}$ long p-type silicon micropillar arrays, with a resistivity of $15-25 \Omega \cdot \mathrm{cm}$ and the BHNO solution described above. Figure 2 shows SEMs of the different stages, and the initial stages and evolution of the tube formation are presented in figure 2(a). After $10 \mathrm{~min}$, the tube formation process is initiated in the central part of the top of the micropillar. This is followed by the formation of additional pores which merge together over the duration of the etching. After about $70 \mathrm{~min}$, a hole has been drilled through the center of the pillar turning it into a tube. The process continues for the following $50 \mathrm{~min}$, widening the hole and becoming almost symmetric. An overview of the arrays before and after $150 \mathrm{~min}$ of etching is presented in figure 2(b). The process occurs homogeneously over the whole array. The outer diameter of the tube does not vary with the etching time, which is related to the doping level of the $\mathrm{Si}$ and the consequent thickness of the SCR, as will be elucidated further down the manuscript.

In order to further explore the mechanisms of tube formation, we have investigated the effect of the $\mathrm{pH}$ of the solution and the doping level of the Si. We used Si pillars with three different doping levels: $10^{17}, 5 \times 10^{15}$ and $5 \times 10^{14} \mathrm{~cm}^{-3}$, as determined by the respective resistivity $0.1-0.5 \Omega \cdot \mathrm{cm}, 1-10$ $\Omega \cdot \mathrm{cm}$ and $15-25 \Omega \cdot \mathrm{cm}$. All these structures were etched at $\mathrm{pH} 0$ and 5, respectively, with the HNO and BHNO solution. The representative SEMs of the structures obtained under the different conditions are shown in figure 3. The SEMs resulting from the etching at $\mathrm{pH}=0$, figures $3(\mathrm{a})-(\mathrm{c})$, were obtained after only $5 \mathrm{~min}$ of etching. The SEM of the structures obtained with $\mathrm{pH}=5$ was performed after $150 \mathrm{~min}$ of etching. We note that the substrate and solution conditions affect the etching speeds directly. Different etching speeds as a function of $\mathrm{pH}$ and electrolyte composition have been reported in the past and will be explained later [46, 47]. For some of the conditions (especially figures 3(d) and (e)) the tubes become faceted. We attribute this to the different dissolution activation energies for the different crystalline planes. These depend both on the doping concentration [59] and $\mathrm{pH}[46,47,60,61]$, which explains why faceting was only clearly observed in some of the conditions.

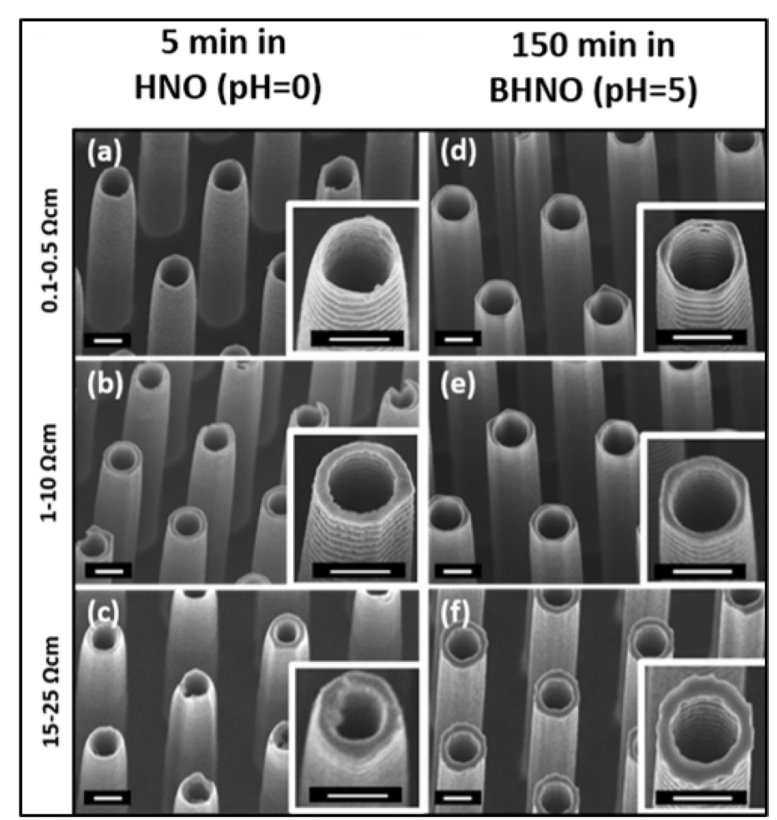

Figure 3. Silicon tubes formed by stain etching (a)-(c) in the HNO solution at $\mathrm{pH} 0$ for $5 \mathrm{~min}$ and (d)-(f) in the BHNO solution at $\mathrm{pH}$ 5 for $150 \mathrm{~min}$. Different doping levels of Si were used, defined by the resistivity values for each row respectively; the scale bar for all images is $2 \mu \mathrm{m}$.

So far, we have given a phenomenological illustration of the tube formation as a function of the $\mathrm{pH}$ and doping concentration. In the following, we move a step forward to model the tube formation in a more precise manner. For this, we consider the formation of the SCR at the semiconductor/ electrolyte interface. It will be shown that at higher depletion lengths, the pillar's outer surface helps to redirect the etching to other regions - e.g. inside the pillar forming a tube-and thus preserve the external walls $[46,47]$. In the case of highaspect-ratio silicon micropillars coming into contact with the electrolyte, a depletion layer is formed at the curved surface, in the same way as in the case of planar geometry. In order to explain the effect of geometry on SCR formation at the semiconductor/electrolyte interface, charge depletion region widths have been derived for the representative geometries. Cylindrical and spherical concave and convex morphologies were chosen, both in the tubular and spherical geometry. The results of these configurations will help us understand the evolution of the SCR at the pillar surfaces and inside the tube once formed. Comparison with the planar configuration will also help us understand where it is more preferable to start dissolution. The geometries used are sketched above the plot in figure 4. Solutions of the Poisson equation in silicon near the interface for the spherical and cylindrical convex and concave geometries were calculated. The resulting equations are a function of the SCR width for planar surfaces, $W_{\mathrm{P}}$. Derivations in reference to the $W_{\mathrm{P}}$ were obtained following the work by Luscombe and Frenzen [62], Zhang [63] and Nersesyan and Petrosyan [64]. For simplicity, the full depletion approximation was used [65]. As boundary conditions, we fixed a finite potential at the surface, and a vanishing potential and electric field at the boundary of the depletion region. 

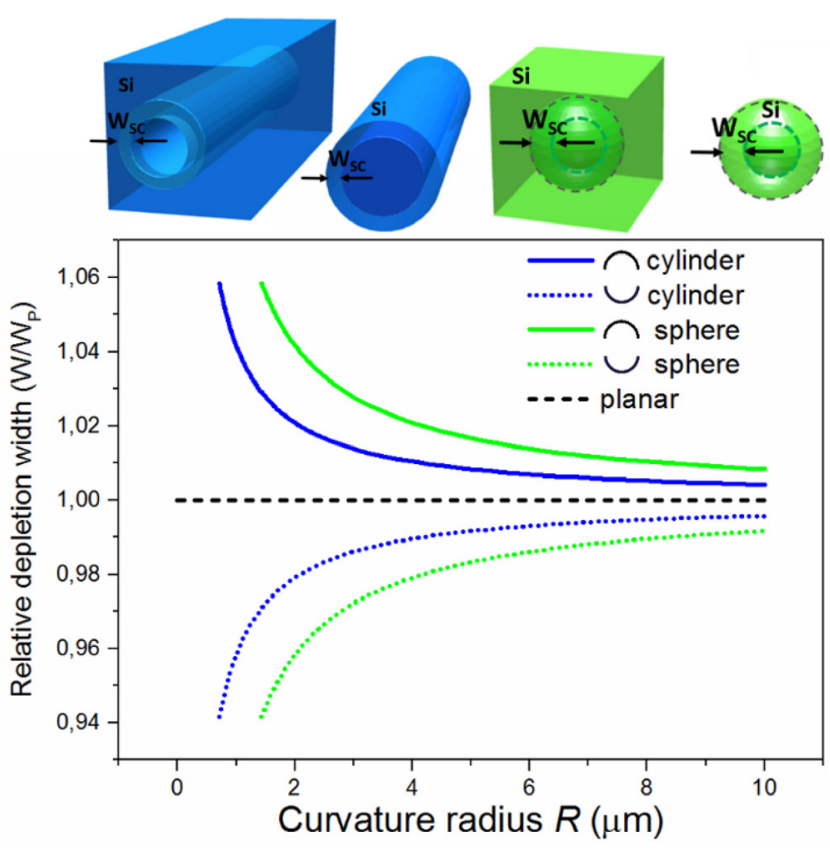

Figure 4. Dependence of the depletion region widths for cylindrical and spherical convex and concave geometries on the curvature radius $R$, compared to a semiconductor with a depletion region width of $W_{\mathrm{P}}$ in the planar geometry, according to the equations given in table 1 . The depletion region widths are given relative to the depletion width of a planar semiconductor, $W_{\mathrm{P}}$. Above the plot are the schematics of the considered geometries: concave and convex cylindrical (in blue) and concave and convex spherical (in green). The main equations describing the width of the SCR for the different geometries are summarized in table 1; for the derivation of the equations please see SI 4.

Table 1. Expressions of the depletion region widths for spherical and cylindrical convex and concave geometries on the curvature radius $R$. $W_{\mathrm{Scv}}, W_{\mathrm{Scc}}, W_{\mathrm{Ccc}}$ and $W_{\mathrm{Ccv}}$ stand for the depletion widths in the spherical convex, spherical concave, cylindrical concave and cylindrical convex geometries, while $W_{\mathrm{P}}$ stands for the depletion width of the planar semiconductor under the same conditions.

\begin{tabular}{ll}
\hline Geometry & Depletion width \\
\hline Spherical convex & $W_{\mathrm{Scv}}=W_{\mathrm{P}}\left(1+\frac{W_{\mathrm{P}}}{3 R}+\frac{5 W_{\mathrm{P}}^{2}}{18 R^{2}}+\ldots\right)$ \\
Spherical concave & $W_{\mathrm{Scc}}=W_{\mathrm{P}}\left(1-\frac{W_{\mathrm{P}}}{3 R}+\frac{5 W_{\mathrm{P}}^{2}}{18 R^{2}}+\ldots\right)$ \\
Cylindrical convex & $W_{\mathrm{Ccv}}=W_{\mathrm{P}}\left(1+\frac{W_{\mathrm{P}}}{6 R}+\frac{W_{\mathrm{P}}^{2}}{9 R^{2}}+\ldots\right)$ \\
Cylindrical concave & $W_{\mathrm{Ccc}}=W_{\mathrm{P}}\left(1-\frac{W_{\mathrm{P}}}{6 R}+\frac{W_{\mathrm{P}}^{2}}{9 R^{2}}+\ldots\right)$ \\
\hline
\end{tabular}

In figure 4 we show the relative difference in width of the SCR for four different curved geometries, in relation to the width of the SCR for a planar semiconductor. We have calculated the SCR widths for a semiconductor sphere and infinite cylinder (the convex case), as well as for a spherical void inside a semiconductor, and for an infinite cylindrical void inside a semiconductor (concave case). The results show that in the case of concave geometries, the width of the SCR is less than in the case of the planar semiconductor, while in the case

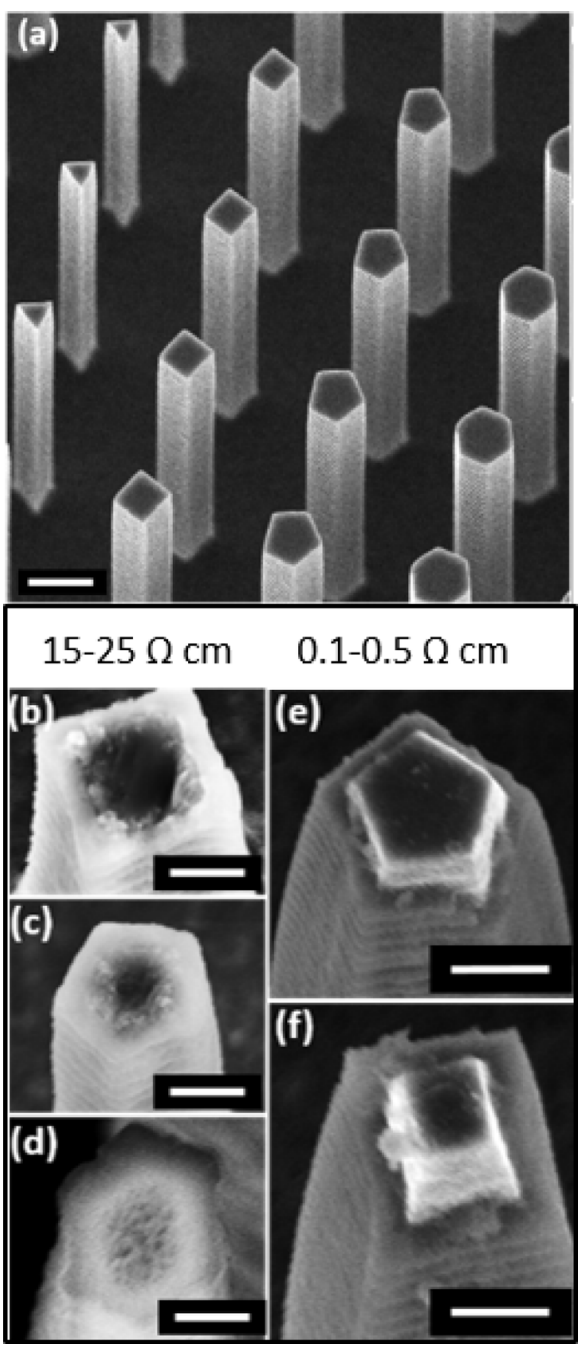

Figure 5. The SEM of arrays with Si pillars with different crosssections (a) and the corresponding morphology after $6 \mathrm{~min}$ of etching in the HNO solution (b)-(f); the scale bars are $10 \mu \mathrm{m}$ wide.

of convex geometries, the SCR widths are larger. Due to the relative nature of the calculations, the results are applicable to any semiconductor in the depletion regime, regardless of the doping level or width of the SCR in the planar geometry. The values of SCR are highest for convex geometries and lowest for concave geometries. While the absolute difference is not very large, this becomes highly relevant for structures approaching sizes of a few micrometer and below. The width of the SCR is smaller in the planar than in the convex case. As a consequence, the dissolution process should occur more preferably at the top flat facet of the pillar with respect to the curved side surfaces. In addition, once a small pore is formed, the surface becomes concave and the SCR is reduced thereby increasing the dissolution rate. This is in agreement with the time evolution of pore formation presented in figure 1. In the case of highly doped silicon where SCR is very thin, etching can also occur on the side facets. This etching is faster for the preferred crystalline directions. As a consequence, facets with the lowest etching velocity develop. The sharp corners intersecting the facets exhibit a higher SCR due to their strongly 


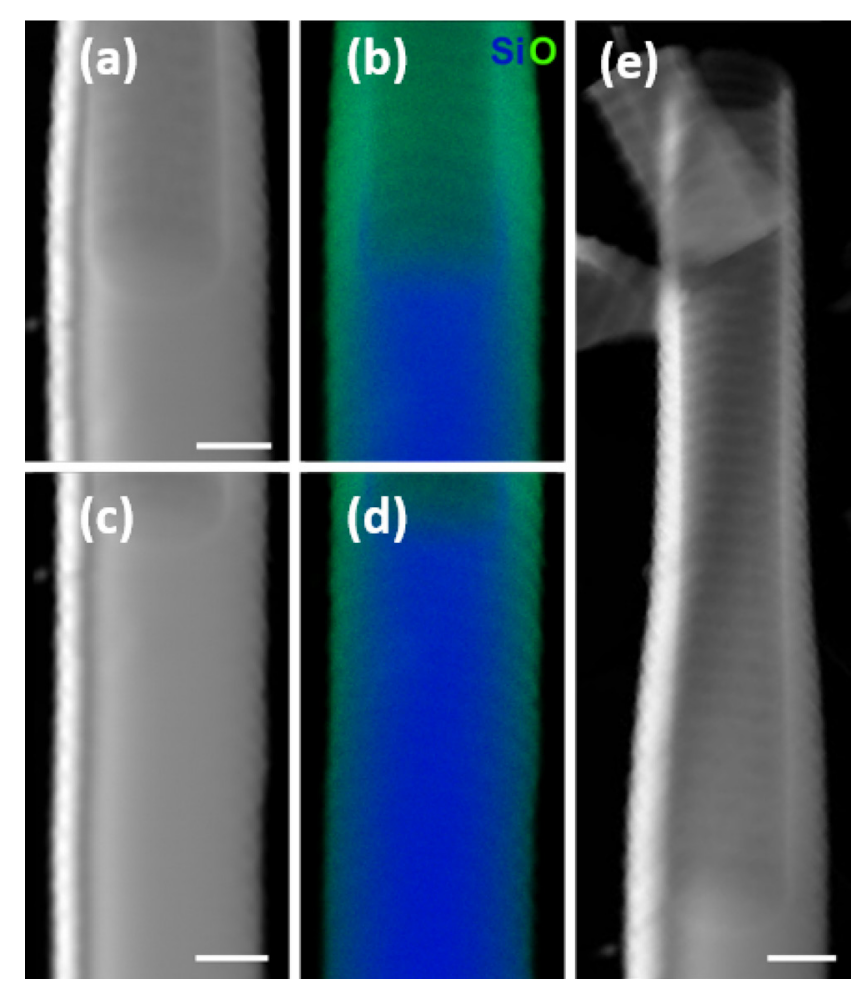

Figure 6. (a)-(d) HAADF and EDX compositional maps of the segments of the tube fabricated by 5 min etching of $35 \mu \mathrm{m}$ long silicon pillars with a resistivity of $0.1-0.5 \Omega \cdot \mathrm{cm}$ in HNO solution, (e) an HAADF image of the drilled part of the same tube from (b)-(e). Both effects are visible with drilling up to half the height and lateral etching; the scale bar is $1 \mu \mathrm{m}$ wide.

reduced radius, and therefore remain protected. This lateral etching and faceting of the pillars is clearly visible in figures 3(d) and (e) as thinning of the sidewalls towards the top of the tube.

In order to further elaborate on the geometry dependence of tube formation, we investigated the etching of pillars with other cross-section geometries: triangular, square, pentagonal and hexagonal. A representative SEM of the array of the structure is shown in figure 5(a). We used wafers with the highest and lowest resistivity, $15-25 \Omega \cdot \mathrm{cm}$ and $0.1-0.5 \Omega \cdot \mathrm{cm}$, in order to validate the role of the geometry and depth of the SCR on the etching process. Figures 5(b)-(f) show the SEM of the different pillars shapes after etching for $6 \mathrm{~min}$ in the HNO solution. We distinguish two different behaviors depending on the doping. For the highest resistivity (figures 5(b)-(d)) the SCR is thick so that etching is preferential on the top of the pillar but also on the flat facets. Etching proceeds homogeneously through the whole structure. The etching at the top of the pillar is slow, compared to the structures obtained with the cylindrical pillars. For the lowest resistivity (figures 5(e) and (f)), the SCR is so thin that etching occurs homogeneously through the whole structure. Interestingly, we observe that the tops of the pillars do not seem to have been attacked. We attribute this to the facet selectivity of the reaction: e.g. (100) versus (110).

In order to elucidate the structure and composition of the porous structures, transmission electron microscopy (TEM) was performed. TEM analysis was performed on the Si pillars with the lowest resistivity used $(0.1-0.5 \Omega \cdot \mathrm{cm})$ and treated for $5 \mathrm{~min}$ in the HNO solution. In order to elucidate the structure and composition of the porous structures, scanning transmission electron microscopy (STEM) and energy dispersive X-ray spectroscopy (EDX) was performed with an FEI Tecnai Osiris microscope operated at $200 \mathrm{kV}$. The pillars were broken off the substrate and transferred by softly wiping the sample with a TEM grid. Figure 6(a) shows an STEM high angular annular dark field (HAADF) image of a pillar: a solid pillar and a hollow tube can clearly be distinguished. Figure 6(b) presents the corresponding compositional EDX map, indicating silicon in blue and oxygen in green. We observe that the hollow tube is fully oxidized, while the solid part of the pillar shows an oxidized surface and significantly lower oxygen content at the center. Figures 6(c) and (d) show an HAADF image and EDX map of the same pillar just below the beginning of the tube. We observe that the oxygen content at the surface of the pillar increases towards the top. As the oxidation rate of porous silicon is higher for a higher porosity, this means that lateral etching is more pronounced on the side walls of the tubular part. Figure 6(e) shows an HAADF image of the full tube, indicating a depth of approximately $11 \mu \mathrm{m}$.

In addition, we characterized the tube depth for the sample with the lowest doping level used (resistivity 15-25 $\Omega \cdot \mathrm{cm}$ ), treated for $150 \mathrm{~min}$ in the BHNO. Figure 7(a) shows a typical SEM of the array cross-section prepared by ion beam thinning. We find that the depth of the tube is about half of the initial pillar height. This result is similar to what we report in figure 6(e); it seems that the tube does not progress to the bottom of the pillar. Tube progression may stop for two possible reasons: first, the SCR at the bottom of the pillar may be influenced or screened by the bulk substrate; secondly, the transport of the species necessary for stain etching may be limited for high-aspect-ratio tubes and may deplete the solution inside. This could slow down or completely stop the advance of tube formation [66].

To try to understand if the stopping of tube progression can be assigned to any one of these options, a series of stain etching in both HNO and BHNO solutions was performed on short pillars $(12-18 \mu \mathrm{m})$ for different times and for all three doping levels reported here. Figure 7(b) shows an SEM of short $(12 \mu \mathrm{m})$ silicon pillars with a resistivity of $15-25 \Omega \cdot \mathrm{cm}$ in the BHNO solution. All the parameters are the same as for the sample in figure 7(a), except for the height of the pillars. No tube formation or significant lateral etching was detected. Tube formation was not observed-not even after $24 \mathrm{~h}$ of etching (figure 7 (c)) - but we found that the pillars were shortened and etched laterally. This result indicates that the propagation of the tubes is not limited exclusively by the reaction-induced depletion of the solution species, but it is probably also related to the change in hole distribution due to the vicinity of the bulk of the substrate. The result of the same experiment for the highest doping level used $(0.1-0.5 \Omega \cdot \mathrm{cm})$ is presented in SI 2. 

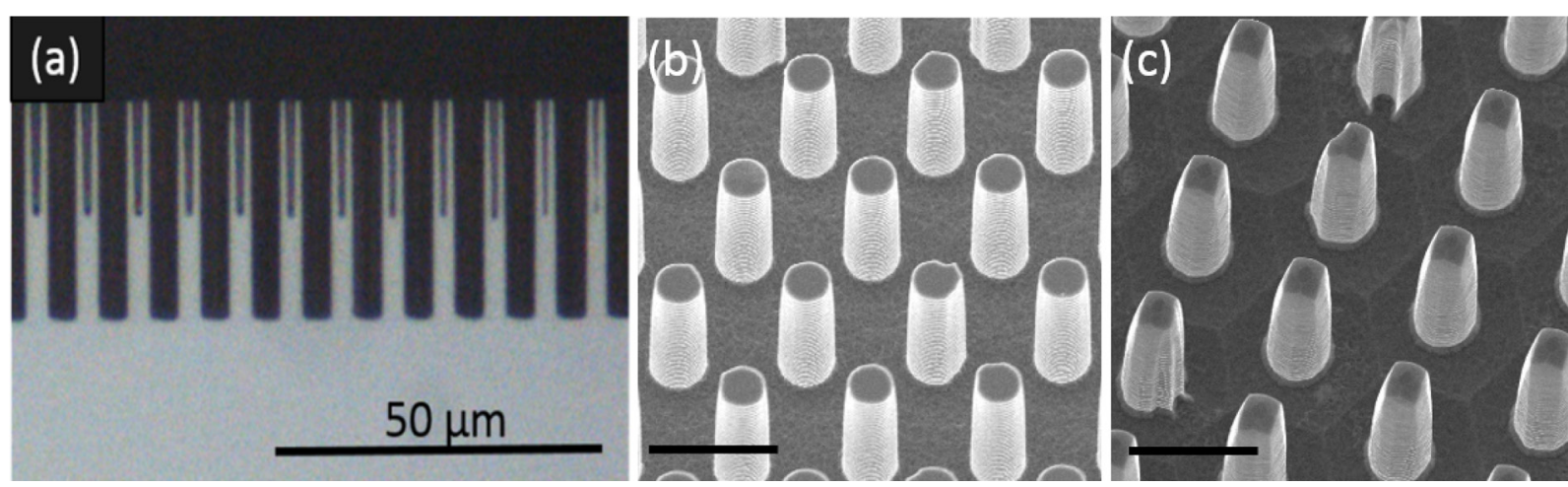

Figure 7. (a) The cross-section prepared by ion beam thinning of the tube fabricated by $150 \mathrm{~min}$ etching of $35 \mu \mathrm{m}$ long silicon pillars with a resistivity of $15-25 \Omega \cdot \mathrm{cm}$ in the BHNO solution; the drilling goes half way through. (b) SEMs of short (12 $\mu \mathrm{m})$ silicon pillars with a resistivity of $15-25 \Omega \cdot \mathrm{cm}$ in the BHNO solution. In this case, drilling never occurred; there is no evidence of significant lateral etching. (c) The SEMs of short $(12 \mu \mathrm{m})$ silicon pillars with a resistivity of $15-25 \Omega \cdot \mathrm{cm}$ in the BHNO solution for $24 \mathrm{~h}$. There is still no evidence of tube formation-just lateral etching and shortening of the pillars was observed. The scale bar for (b) and (c) is $5 \mu \mathrm{m}$ and the tilt angle $20^{\circ}$.

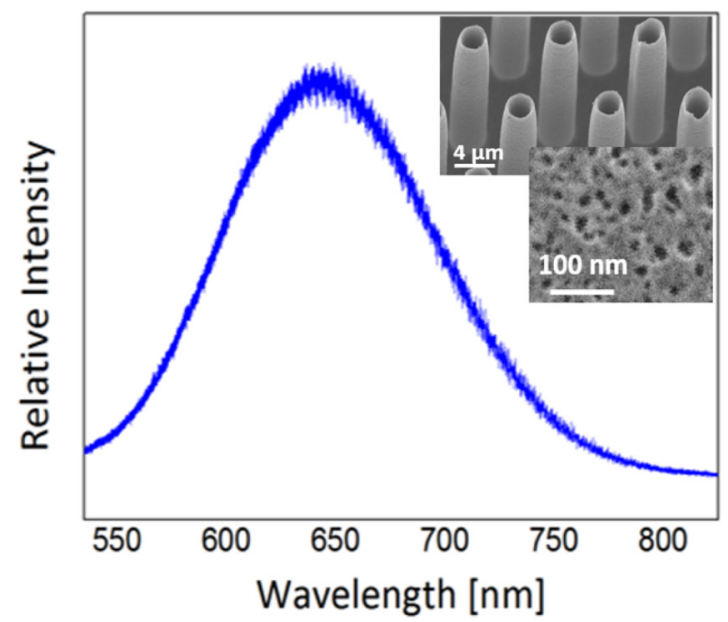

Figure 8. The photoluminescence spectrum of porous Si tubes fabricated by the two-step stain etching process (BHNO and HVO).

\section{Optical characterization}

For the optical characterization of the Si tube arrays, we used photoluminescence (PL) and CL spectroscopy. The samples used in the optical characterization and device fabrication were prepared in a two-step process. We started etching the pillars in the BHNO solution to create highly homogeneous tube arrays. We then exposed the structures to the HVO solution to form a more uniform porous layer, see [24, 44, 67, 67]. We used silicon pillars with the highest amount of doping, since according to Nahidi, the PL signal of the corresponding porous silicon is the strongest in this case [68].

Figure 8 shows a typical micro-PL spectrum of these samples. The excitation source is the $514.5 \mathrm{~nm}$ line of an Argon-ion laser. A power of $1 \mathrm{~mW}$ is projected onto the sample by a $100 \times$ microscope objective. We observe a broad band centered at around $650 \mathrm{~nm}$. Similar PL spectra are observed in most porous silicon samples, regardless of the preparation. The inset in the figure shows part of the microtube array and a zoom of the surface of the single microtube in order to present the porosity level. The two most commonly encountered luminescence emission bands reported for porous silicon are a)

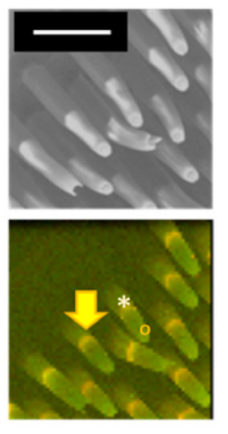

b)

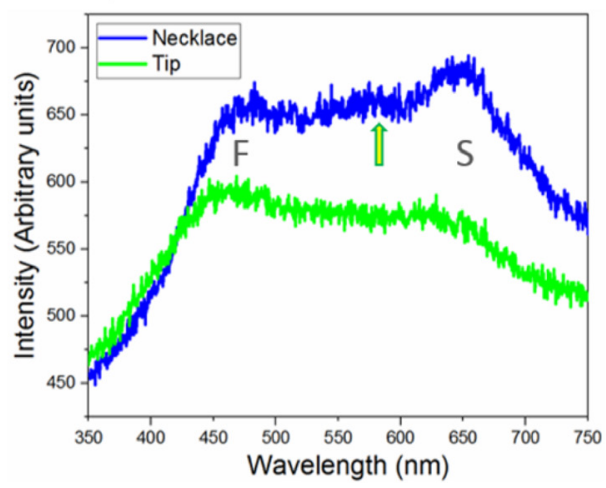

Figure 9. (a) SEM (top) and CL map (below) of silicon micropillar arrays covered by luminescent porous silicon. In the CL maps, red and green correspond to the emission centered at $650 \mathrm{~nm}$ and $466 \mathrm{~nm}$, respectively. The oxidation-related $\mathrm{F}$ band emission around $450 \mathrm{~nm}$ is especially bright at half the height of the each pillar (necklace), while the quantum-confinement-related luminescence in the $\mathrm{S}$ band is dominant near the top of the porous pillars; the scale bar is $5 \mu \mathrm{m}$. (b) The CL spectra acquired in two different positions on the tube, in which the green curve corresponds to the spectrum from the tube tip/wall and the blue curve corresponds to the spectrum taken from the interface at the so-called necklace.

the 'slow' or 'S' red band, and the 'fast' or ' $F$ ' blue band. The $\mathrm{S}$ band is centered between 600 and $850 \mathrm{~nm}$, and it decays on the $10-100 \mathrm{~ms}$ scale. The origin of this luminescence is usually explained as radiative recombination from spatially confined excitons in the silicon nanocrystal. The F band luminescence, usually centered between 420 and $500 \mathrm{~nm}$, decays on the nanosecond time scale. This can be observed on porous silicon samples aged in air or intentionally oxidized, and is thought to originate from structural defects in the silicon nanocrystal oxide shell, or from the luminescence of very small silicon nanocrystals [19, 39, 69-72]. Other PL bands have been previously reported for porous silicon: the so-called UV band (centered around $350 \mathrm{~nm}$ ), and the R band (ranging from $1100-1500 \mathrm{~nm}$ ) [59].

We proceed now with the CL characterization of the tubes. With respect to the PL, scanning electron microscopy cathodoluminescence (SEM-CL) can excite luminescence with a much 


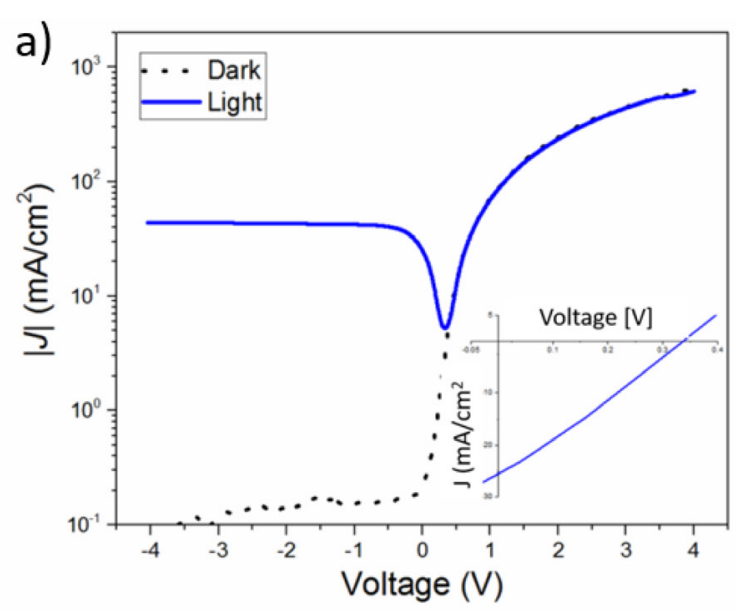

b)

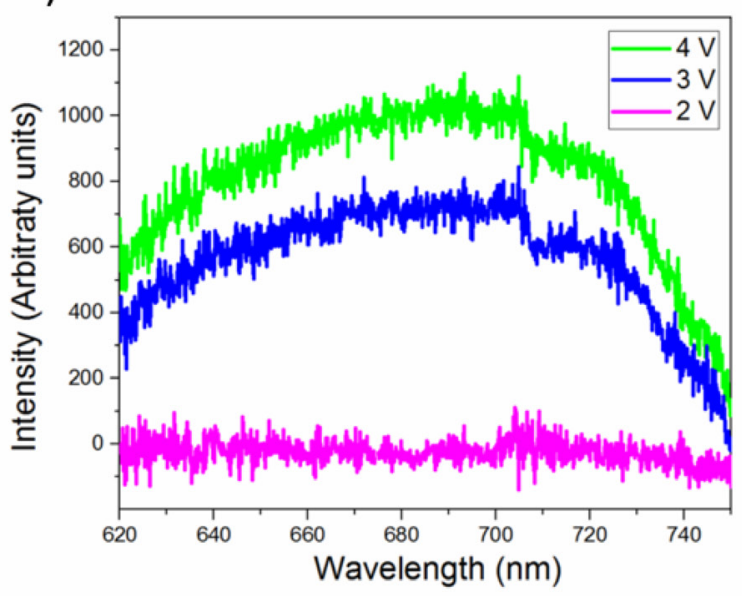

Figure 10. (a) The absolute $J-V$ characteristics for the Si-tube array device taken in the dark and under illumination by a solar simulator set at an irradiance of $100 \mathrm{~mW} \mathrm{~cm}{ }^{-2}$. The inset shows the $J-V$ curve between $0 \mathrm{~V}$ and $0.4 \mathrm{~V}$, outlining the $V_{\mathrm{OC}}$ and $J_{\mathrm{SC}}$. (b) Electroluminescence spectra of the same device at different operation voltages showing wide-band light emission.

higher spatial resolution. The SEM-CL e-beam probe is a few nanometers in diameter compared to at least a few hundred nanometers for the laser spot in micro-PL. It is also worth noticing that CL offers a way of accessing excitation energies beyond UV. To the best of our knowledge, CL studies of porous silicon are rare. Figure 9 presents the results from the CL studies, performed with an Attolight SEM-CL microscope at an acceleration voltage of $7 \mathrm{kV}$. In figure 9(a) we show the SEM of the investigated region along with the mapping of the CL signal. We observe light emission from the sidewall regions, where there is an oxide. In the arbitrary color-coding of the CL map, red and green correspond to the emission centered at $650 \mathrm{~nm}$ and $446 \mathrm{~nm}$ respectively. This emphasizes the redshift of the emission at the bottom of the tubes, which corresponds to half the height of the full pillar. This position is indicated by a yellow arrow on one of the pillars in figure 9(a). This region has the appearance of a necklace and we will thereafter refer to it thus. The spectra from the top of the tube and from the necklace region (marked by ${ }^{\circ}$ and ${ }^{*}$ respectively on the CL map) are presented in figure 9(b). With respect to the PL measurements, the electron excitation allows us to access higher energy emissions. In fact, both spectra present two bands that can be identified as the $\mathrm{F}$ and $\mathrm{S}$ bands. The intensity ratio between the two types of emission changes with the position on the pillar. The spectrum obtained at the necklace has a higher S/F intensity ratio compared to the spectrum obtained at the tube tip/walls. The $\mathrm{F}$ band is related to the defects at the silicon/silicon oxide interface $[69,70,73]$. The necklace region corresponds to the area where the tube is actively forming. Oxidization may still be incomplete, which could account for a higher presence of defects and an increased intensity of the F band. Similarly, the CL emission of both bands dims while moving away from the tube, especially below the necklace. This could be related to the lower presence of oxide towards the microtube bottom, as shown by the EDX mappings in figures 6(b)-(d). Between the F and $\mathrm{S}$ bands there is also a possible third band at $580 \mathrm{~nm}$, which is marked with an arrow in the blue spectrum in figure 9(b). One possible explanation could be the blueshift of part of the $\mathrm{S}$ band due to quantum confinement in the silicon nanoparticles [30].

\section{Demonstration of 3D light emitting device}

In this part, we demonstrate the functionality of these structures by fabricating a light emitting diode. For this, we evaporated an aluminum Ohmic contact onto the back side of the sample and sputtered $100 \mathrm{~nm}$ of ITO onto the top of the porous silicon side as a transparent electrode; for more details see SI 1. The size of the active device was $7.15 \times 7.75 \mathrm{~mm}^{2}$, which is the area that we used for the calculation of the current density, although the real effective area of the diode surface is higher. The current-voltage diode characteristics of the device are shown in figure 10(a). We show both the device characteristics in the dark and under AM 1.5 G illumination conditions. Under solar illumination we obtain a $V_{\mathrm{OC}}$ of $0.34 \mathrm{~V}$, a $J_{\mathrm{SC}}$ of $25 \mathrm{~mA} \mathrm{~cm}{ }^{-2}$ and a fill factor of $27 \%$. Fitting the first quadrant of the current-voltage characteristics gives an ideality factor of 2.5 (for more details see SI 3). We obtain a power conversion efficiency of $2.3 \%$. The rectifying ratio of the solar cell in the dark between -4 and $4 \mathrm{~V}$ was $10^{4}$. The relatively high reverse currents, relatively low fill factor, $V_{\mathrm{OC}}$ and PCE are indicative of the high surface recombination of the photogenerated charge carriers. Dangling bonds in the porous silicon provide the defect levels in the bandgap of silicon and should be mainly responsible for the observed charge recombination [73-78].

We measured the electroluminescence of the device by applying a forward bias, and the resulting spectra are shown in figure 10 (b). We observed wide band electroluminescence centered at around $690 \mathrm{~nm}$. EL emission starts to be detected for an applied voltage of $3 \mathrm{~V}$ and it further increases at higher bias till $4 \mathrm{~V}$; for higher voltages the emission is quenched. The emission is recovered when the voltage is further reduced to the 3-4 $\mathrm{V}$ range. The reference planar devices prepared under the same conditions did not show any measurable EL: the EL device was stable during the whole length of the measurements lasting a few hours. 


\section{Conclusions}

In conclusion, we have investigated the electroless formation of porous silicon tubes, organized in an ordered manner. By looking at tube formation as a function of the pillar size, geometry and doping, we have pointed out the main formation mechanisms. In particular, we have shown how the 3D geometry creates an inhomogeneous SCR that provides paths of least resistance for an inhomogeneous etching. The optical properties of the porous structures are in agreement with the characteristics of Si nanocrystals and porous silicon, as shown by the photo and CL studies. Finally, we demonstrate the functionality of our 3D structures by fabricating a diode structure. The diode can work both as a solar cell or detector and as a light emitting diode. This work shows how silicon microtube arrays can be used for optoelectronics, with possible further advantages in photo electrochemistry, battery electrodes and supercapacitors.

\section{Acknowledgments}

We acknowledge P Lazić for the valuable discussions, CIME for access to the microscopes and Danièle Laub for the fabrication of the cross-sections.

\section{Author contributions}

The manuscript was written through the contributions of all the authors. All authors have given approval to the final version of the manuscript.

\section{Funding sources}

This work has been partially supported by the Croatian Science Foundation under the project (IP-2014-09-7046). JV, HP, FA and AFiM thank funding support from the SNF through the NCCR-QSIT, and Nanotera Synergy, ITN projects from FP7 Nanoembrace and H2020 Indeed.

\section{References}

[1] Lockwood D J 1998 Light Emission in Silicon: From Physics to Devices (New York: Academic)

[2] Fauchet P M 2004 Monolithic silicon light sources Silicon Photonics (Berlin: Springer) pp 177-98

[3] Vivien L and Pavesi L 2013 Handbook of Silicon Photonics (London: Taylor and Francis)

[4] King O and Hall D G 1994 Impurity-related photoluminescence from silicon at room temperature Phys. Rev. B 50 10661-5

[5] Fiory A T and Ravindra N M 2003 Light emission from silicon: some perspectives and applications J. Electron. Mater. 32 1043-51

[6] Deboy G and Kolzer J 1994 Fundamentals of light emission from silicon devices Semicond. Sci. Technol. 9 1017-32
[7] Jun Y C, Briggs R M, Atwater H A and Brongersma M L 2009 Broadband enhancement of light emission in silicon slot waveguides Opt. Express 17 7479-90

[8] Gelloz B, Kojima A and Koshida N 2005 Highly efficient and stable luminescence of nanocrystalline porous silicon treated by high-pressure water vapor annealing Appl. Phys. Lett. 8731107

[9] Gelloz B and Koshida N 2005 Mechanism of a remarkable enhancement in the light emission from nanocrystalline porous silicon annealed in high-pressure water vapor J. Appl. Phys. 98123509

[10] Gelloz B 2014 Electroluminescence of porous silicon Handbook of Porous Silicon (Cham: Springer) pp 1-11

[11] Lee B G, Luo J-W, Neale N R, Beard M C, Hiller D, Zacharias M, Stradins P and Zunger A 2016 Quasi-direct optical transitions in silicon nanocrystals with intensity exceeding the bulk Nano Lett. 16 1583-9

[12] Zacharias M, Heitmann J, Scholz R, Kahler U, Schmidt M and Bläsing J 2002 Size-controlled highly luminescent silicon nanocrystals: a $\mathrm{SiO} / \mathrm{SiO}_{2}$ superlattice approach Appl. Phys. Lett. 80 661-3

[13] Godefroo S, Hayne M, Jivanescu M, Stesmans A, Zacharias M, Lebedev O I, Van Tendeloo G and Moshchalkov V V 2008 Classification and control of the origin of photoluminescence from Si nanocrystals Nat. Nanotechnol. 3 174-8

[14] Biteen J S, Lewis N S, Atwater H A and Polman A 2004 Size-dependent oxygen-related electronic states in silicon nanocrystals Appl. Phys. Lett. 84 5389-91

[15] Gelloz B and Koshida N 2000 Electroluminescence with high and stable quantum efficiency and low threshold voltage from anodically oxidized thin porous silicon diode $J$. Appl. Phys. 884319

[16] Korotcenkov G 2016 Porous Silicon: From Formation to Application: Formation and Properties, Volume One: Formation and Properties (Boca Raton, FL: CRC Press)

[17] Uhlir A 1956 Electrolytic shaping of germanium and silicon Bell Syst. Tech. J. 35 333-47

[18] Canham L T 1990 Silicon quantum wire array fabrication by electrochemical and chemical dissolution of wafers Appl. Phys. Lett. 571046

[19] Canham L 2014 Handbook of Porous Silicon (Berlin: Springer)

[20] Lehmann V and Gösele U 1991 Porous silicon formation: a quantum wire effect Appl. Phys. Lett. 58 856-8

[21] Lehmann V and Gösele U 1992 Porous silicon: quantum sponge structures grown via a self-adjusting etching process Adv. Mater. 4 114-6

[22] Sailor M J 2012 Porous Silicon in Practice: Preparation, Characterization and Applications (New York: Wiley)

[23] Kolasinski K W 2015 Electron transfer during metal-assisted and stain etching of silicon Semicond. Sci. Technol. 3114002

[24] Kolasinski K W and Barclay W B 2013 Stain etching of silicon with and without the aid of metal catalysts ECS Trans. 50 25-30

[25] Vázsonyi É, Szilágyi E, Petrik P, Horváth Z, Lohner T, Fried M and Jalsovszky G 2001 Porous silicon formation by stain etching Thin Solid Films 388 295-302

[26] González-Díaz B, Guerrero-Lemus R, Marrero N, HernándezRodríguez C, Ben-Hander F A and Martínez-Duart J M 2006 Anisotropic textured silicon obtained by stain-etching at low etching rates J. Phys. D: Appl. Phys. 39 631-4

[27] Parbukov A, Beklemyshev V, Gontar V, Makhonin I, Gavrilov S and Bayliss S 2001 The production of a novel stain-etched porous silicon, metallization of the porous surface and application in hydrocarbon sensors Mater. Sci. Eng. C 15 121-3 
[28] Kolasinski K W and Yadlovskiy J 2011 Stain etching of silicon with $\mathrm{V}_{2} \mathrm{O}_{5}$ Phys. Status Solidi 8 1749-53

[29] Ünal B, Parbukov A N and Bayliss S C 2001 Photovoltaic properties of a novel stain etched porous silicon and its application in photosensitive devices Opt. Mater. 17 79-82

[30] Ledoux G, Gong J, Huisken F, Guillois O and Reynaud C 2002 Photoluminescence of size-separated silicon nanocrystals: confirmation of quantum confinement Appl. Phys. Lett. 804834

[31] Tsybeskov L, Duttagupta S P, Hirschman K D and Fauchet P M 1996 Stable and efficient electroluminescence from a porous silicon-based bipolar device Appl. Phys. Lett. 68 2058-60

[32] Canham L T, Loni A, Simons A J, Cox T I and Calcott P D J 1995 Electroluminescent porous silicon device with an external quantum efficiency greater than $0.1 \%$ under $\mathrm{CW}$ operation Electron. Lett. 31 1288-9

[33] Pavesi L, Ceschini M, Mariotto G, Zanghellini E, Bisi O, Anderle M, Calliari L, Fedrizzi M and Fedrizzi L 1994 Spectroscopic investigation of electroluminescent porous silicon J. Appl. Phys. 75 1118-26

[34] Canham L T, Leong W Y, Beale M I J, Cox T I and Taylor L 1992 Efficient visible electroluminescence from highly porous silicon under cathodic bias Appl. Phys. Lett. $612563-5$

[35] Snow P A, Squire E K, Russell P S J and Canham L T 1999 Vapor sensing using the optical properties of porous silicon Bragg mirrors J. Appl. Phys. 86 1781-4

[36] Pancheri L, Oton C J, Gaburro Z, Soncini G and Pavesi L 2003 Very sensitive porous silicon $\mathrm{NO}_{2}$ sensor Sensors Actuators B 89 237-9

[37] Erson R C, Muller R S and Tobias C W 1990 Investigations of porous silicon for vapor sensing Sensors Actuators A 23 835-9

[38] Martínez-Duart J M, Parkhutik V P, Guerrero-Lemus R and Moreno J D 1995 Electroluminescent porous silicon Adv. Mater. 7 226-8

[39] Canham L T 1995 Luminescence bands and their proposed origins in highly porous silicon Phys. Status Solidi 190 9-14

[40] Oguro T, Koyama H, Ozaki T and Koshida N 1997 Mechanism of the visible electroluminescence from metal/ porous silicon/n-Si devices J. Appl. Phys. 81 1407-12

[41] Gelloz B, Nakagawa T and Koshida N 1998 Enhancement of the quantum efficiency and stability of electroluminescence from porous silicon by anodic passivation Appl. Phys. Lett. 73 2021-3

[42] Kolasinski K W 2003 The mechanism of Si etching in fluoride solutions Phys. Chem. Chem. Phys. 5 1270-8

[43] Kolasinski K W and Barclay W B 2013 The stoichiometry of electroless silicon etching in solutions of $\mathrm{V}_{2} \mathrm{O}_{5}$ and $\mathrm{HF}$ Angew. Chem., Int. Ed. 52 6731-4

[44] Dudley M E and Kolasinski K W 2009 Stain etching with $\mathrm{Fe}(\mathrm{III}), \mathrm{V}(\mathrm{V})$, and $\mathrm{Ce}(\mathrm{IV})$ to form microporous silicon Electrochem. Solid-State Lett. 12 D22

[45] Gerischer H 1969 On the role of electrons and holes in surface reactions on semiconductors Surf. Sci. 13 265-78

[46] Huanca D R and Salcedo W J 2014 Mesoporous silicon: a new route to fabricate silicon-based nanotubes Phys. Status Solidi 211 1525-30

[47] Roque D, Yong H and Jaimes W 2015 Silicon microtubes made by immersing macroporous silicon into ammonium fluoride solution Mater. Chem. Phys. $16012-9$

[48] Ling T and Guo L J 2007 A unique resonance mode observed in a prism-coupled micro-tube resonator sensor with superior index sensitivity Opt. Express 15 17424-32

[49] Skryshevsky V, Lysenko V and Livinenko S 2016 Hydrogen generation and storage in porous silicon Porous Silicon: From Formation to Applications: Optoelectronics,
Microelectronics, and Energy Technology Applications vol 3 (Boca Raton, FL: CRC Press) pp 273-95

[50] Golodnitsky D, Strauss E and Ripenbein T 2016 PSi-based supercapacitors Porous Silicon: From Formation to Applications: Optoelectronics, Microelectronics, and Energy Technology Applications vol 3 (Boca Raton, FL: CRC Press) pp 347-74

[51] Gautier G and Korotcenkov G 2016 Porous silicon in micro-fuel cells Porous Silicon: From Formation to Applications: Optoelectronics, Microelectronics, and Energy Technology Applications vol 3 (Boca Raton, FL: CRC Press) pp 249-72

[52] Park M, Kim M G, Joo J, Kim K, Kim J, Ahn S, Cui Y and Cho J 2009 Silicon nanotube battery anodes Nano Lett. $93844-7$

[53] Mu C, Zhao Q, Xu D, Zhuang Q and Shao Y 2007 Silicon nanotube array/gold electrode for direct electrochemistry of cytochrome c J. Phys. Chem. B 111 1491-5

[54] Daldosso N and Pavesi L 2009 Nanosilicon photonics Laser Photon. Rev. 3 508-34

[55] Recio-Sánchez G 2016 PSi-based photonic crystals Porous Silicon: From Formation to Applications: Optoelectronics, Microelectronics, and Energy Technology Applications vol 3 (Boca Raton, FL: CRC Press) pp 51-75

[56] Coffer J L 2016 Mesoporous nanotubes as biomaterials Mesoporous Biomater. 2 33-48

[57] Berthing T, Bonde S, Rostgaard K R, Madsen M H, Sorensen C B, Nygard J and Martinez K L 2012 Cell membrane conformation at vertical nanowire array interface revealed by fluorescence imaging Nanotechnology 238

[58] Berthing T, Sørensen C B, Nygård J and Martinez K L 2009 Applications of nanowire arrays in nanomedicine J. Nanoneurosci. $13-9$

[59] Sailor M J 2011 Porous Silicon in Practice (Weinheim: Wiley)

[60] Zhang X G 2001 Electrochemistry of Silicon and its Oxide (New York: Springer)

[61] Madou M J 2002 MEMS Design and fabrication (Boca Raton, FL: CRC Press)

[62] Luscombe J H and Frenzen C L 2002 Depletion lengths in semiconductor nanostructures Solid State Electron. 46 885-9

[63] Zhang X G 1991 Mechanism of pore formation on n-type silicon J. Electrochem. Soc. 1383750

[64] Nersesyan S R and Petrosyan S G 2012 Depletion length and space charge layer capacitance in doped semiconductor nanosphere Semicond. Sci. Technol. 27125009

[65] Sze S M and Ng K K 2006 Physics of Semiconductor Devices (New York: Wiley)

[66] Kolasinski K W 2010 Charge transfer and nanostructure formation during electroless etching of silicon J. Phys. Chem. C 114 22098-105

[67] Kolasinski K W 2014 Bubbles: a review of their relationship to the formation of thin flms and porous materials Mesoporous Biomater. 1 49-60

[68] Nahidi M and Kolasinski K W 2006 Effects of stain etchant composition on the photoluminescence and morphology of porous silicon J. Electrochem. Soc. $153 \mathrm{C} 19$

[69] Kontkiewicz A J et al 1994 Evidence that blue luminescence of oxidized porous silicon originates from $\mathrm{SiO}_{2}$ Appl. Phys. Lett. 65 1436-8

[70] Cooke D W, Bennett B L, Farnum E H, Hults W L, Sickafus K E, Smith J F, Smith J L, Taylor T N, Tiwari P and Portis A M $1996 \mathrm{SiO}_{x}$ luminescence from light-emitting porous silicon: support for the quantum confinement/ luminescence center model Appl. Phys. Lett. 68 1663-5

[71] Canham L, Loni A, Calcott P D, Simons A, Reeves C, Houlton M, Newey J, Nash K and Cox T 1996 On the origin of blue luminescence arising from atmospheric impregnation of oxidized porous silicon Thin Solid Films 276 112-5 
[72] Valenta J, Fucikova A, Pelant I, Kůsová K, Dohnalová K, Aleknavičius A, Cibulka O, Fojtík A and Kada G 2008 On the origin of the fast photoluminescence band in small silicon nanoparticles New J. Phys. 1073022

[73] Carlos W E and Prokes S M 1995 The EX defect center in porous silicon J. Appl. Phys. 78 2129-31

[74] Hotovy J, Hüpkes J, Böttler W, Marins E, Spiess L, Kups T, Smirnov V, Hotovy I and Kováč J 2013 Sputtered ITO for application in thin-film silicon solar cells: relationship between structural and electrical properties Appl. Surf. Sci. 269 81-7

[75] Yu Q, He H, Gan L and Ye Z 2015 The defect nature of photoluminescence from a porous silicon nanowire array RSC Adv. 5 80526-9
[76] von Bardeleben H J, Stievenard D, Grosman A, Ortega C and Siejka J 1993 Defects in porous p-type Si: an electron-paramagnetic-resonance study Phys. Rev. B 47 10899-902

[77] Prokes S M and Carlos W E 1995 Oxygen defect center red room temperature photoluminescence from freshly etched and oxidized porous silicon $J$. Appl. Phys. 78 2671-4

[78] Gelloz B, Sano H, Boukherroub R, Wayner D D M, Lockwood D J and Koshida N 2003 Stabilization of porous silicon electroluminescence by surface passivation with controlled covalent bonds Appl. Phys. Lett. $832342-4$ 